UTTG-21-93

June 1993

\title{
A Note on Effective String Theory
}

\author{
Shyamoli Chaudhuri and Djordje Minid] \\ Theory Group \\ Department of Physics \\ University of Texas \\ Austin, Texas 78712
}

\begin{abstract}
$\underline{\text { ABSTRACT }}$
Motivated by the possibility of an effective string description for the infrared limit of pure Yang-Mills theory, we present a toy model for an effective theory of random surfaces propagating in a target space of $D>2$. We show that the scaling exponents for the fixed area partition function of the theory are apparently well behaved. We make some observations regarding the usefulness of this toy model.
\end{abstract}

*Address after Sept. 1 1993: ITP, University of California, Santa Barbara, CA 93106; sc@utaphy.bitnet

${ }^{\dagger}$ Address after Sept. 1 1993: Physics Department, City College of the CUNY, New York, NY 10031; minic@utaphy.bitnet 


\section{Introduction}

It is an old proposal that there exists a dual description of pure YangMills theory in terms of a theory of random surfaces [1]. Motivation for this proposal comes from numerous sources, recently reviewed in [2] [3]: (a) successful Regge phenomenology [4], (b) lattice gauge theory, which in the strong coupling limit seemingly leads to a theory of random surfaces [5], (c) the topological form of the large $\mathrm{N}$ perturbative expansion [6], where $\mathrm{N}$ denotes the number of colors, particularly the application of large $\mathrm{N}$ techniques to the case of two-dimensional QCD [7], and, (d) the structure of the QCD loop equations [8]. More recently, an explicit and complete interpretation of the two-dimensional Yang-Mills partition function as a sum over maps has emerged [9], which may have an analogue for four-dimensional Yang-Mills theory.

The Nambu-Goto string, heuristically suggested by all of these approaches, classically weights maps by the exponential of their area. It is nonpolynomial leading to a nonrenormalizable quantum effective action [11]. The classically equivalent, and renormalizable, Polyakov string quantization introduces effective dynamics for the world-sheet graviton in the form of the Liouville mode [13]. This creates two problems for a putative QCD string: the presence of an additional massless scalar increasing the effective number of transverse degrees of freedom to $D-1$ and thereby spoiling Lorentz invariance [10 [11] [12], and secondly, the presence of a tachyonic excitation in the spectrum of the string. The string theory defined by the two dimensional QCD partition function also appears to point towards introducing new degrees of freedom on the world-sheet, perhaps fermionic, so as to completely supress the tachyonic 
excitation (no folds) [9]. This excitation is massless in two dimensions, and kinematically, its transition to a tachyonic state can be seen in the $D=1$ barrier to real scaling exponents for the quantum non-critical string [14] [15].

One could avoid introducing a world-sheet metric altogether but we must then abandon all of the powerful tools provided by conformal field theory. Perhaps this is the correct approach, however, we will show that it is possible to modify the usual quantization procedure so as to overcome these problems, at least kinematically. Our toy model is the simplest possible modification and will unfortunately turn out to define an unphysical theory. Our hope is that some extension of our reasoning will lead to viable noncritical strings which could then be tested against QCD, through, for example, a comparison of their high-temperature behavior [16].

\section{The World-sheet Theory}

We are therefore motivated to introduce additional local degrees of freedom on the world-sheet, respecting space-time Lorentz invariance and, for the reasons described above, world-sheet conformal invariance. However, we will attempt to modify the equivalence between the time-like string coordinate and the Liouville mode in the usual quantization [10], which suggests that we introduce non-trivial couplings of the internal degrees of freedom to the world-sheet gravity. This will also alter the kinematic scaling behavior

of the string and allow us to avoid the "strong gravity" $(D>1)$ sector of the usual non-critical string. Since we envision infrared effective Yang-Mills strings built out of pure glue we would like our internal degrees of freedom to carry a color index, which naturally suggests a Kac-Moody structure on 
the world-sheet.1

In this paper, we will choose the simplest possible option for the new coupling between gravity and the internal degrees of freedom, which has the virtue of leaving the resulting (and, as will turn out, unphysical) theory completely calculable. Note that more sophisticated modifications, such as extrinsic geometry, can also be represented, at least kinematically, by additional WZNW-type fields on the world-sheet [18].

We are thus led to the following action for our model string theory

$$
S=S_{m}+S_{\text {int }},
$$

where

$$
S_{m}=\int d^{2} \sigma \sqrt{g} g^{a b}\left(\partial_{a} \vec{X} \partial_{b} \vec{X}+\sum_{i} \partial_{a} \phi_{i} \partial_{b} \phi_{i}\right)
$$

and

$$
S_{i n t}=\sum_{i} B_{i} \int d^{2} \sigma \sqrt{g} R \phi_{i}
$$

where $\vec{X}$ denotes a set of $C$ flat space-time coordinates, the operators $\partial \phi_{i}, i=$ $1, \cdots N$, are the maximally commuting subset of generators of the $G^{(k=1)} \mathrm{Kac}-$ Moody algebra and the $\phi_{i}$ are the corresponding Kac-Frenkel-Segal bosons [19]. The restriction to the maximally commuting generators is suggested by the intrinsic $U(1)$ symmetry of the Liouville field theory. More general couplings may be possible. Here $g^{a b}$ and $R$ denote the world-sheet metric and scalar curvature respectively.

Note that in conformal gauge,

$$
g^{a b}=e^{\eta} \hat{g}^{a b},
$$

\footnotetext{
${ }^{1}$ This picture perhaps, seems even more natural if we recall the known relation between the classical Yang-Mills theory and classical chiral theory formulated on the "space" of Wilson loops [17.
} 
where $\eta$ denotes the Weyl mode and $\hat{g}^{a b}$ is the fiducial metric, one immediately generates an interaction term coupling the Weyl factor and the $U(1)$ 's of the form

$$
S_{i n t}=\sum_{i} B_{i} \int d^{2} \sigma \sqrt{\hat{g}}\left(\hat{R}-\hat{\nabla}^{a} \eta \hat{\nabla}_{a}\right) \phi_{i}
$$

Here the curvature scalar and covariant derivatives are defined with respect to the fiducial metric.

The partition function of the theory reads

$$
Z=\int \frac{\mathcal{D} g \mathcal{D} \Phi_{\text {matter }}}{\operatorname{volume}(\text { Diff })} e^{-S},
$$

with $\Phi_{\text {matter }}$ denotes all of the matter fields introduced above. Furthermore the reparametrization invariant measure for the path integration is defined by

$$
|\delta \vec{X}|_{g}^{2}=\int d^{2} \sigma \sqrt{g}(\delta \vec{X})^{2}
$$

and

$$
|\delta g|_{g}^{2}=\int d^{2} \sigma \sqrt{g}\left(g^{a b} g^{c d}+\mathcal{C} g^{a c} g^{b d}\right) \delta g_{a c} \delta g_{b d},
$$

with $\mathcal{C}$ an arbitrary constant, usually set equal to zero. In conformal gauge, $g^{a b}=e^{\eta} \hat{g}^{a b}$, shifting to the fiducial metric results in induced dynamics for the Weyl factor:

$$
\mathcal{D}_{g} \Phi_{\text {matter }}=\mathcal{D}_{\hat{g}} \Phi_{\text {matter }} \exp \left(\frac{c_{m}}{48 \pi} S_{L}\right)
$$

where $c_{m}$ denotes the total central charge of the matter sector, and

$$
S_{L}=\int d^{2} \sigma \sqrt{\hat{g}}\left(\frac{1}{2} \hat{g}^{a b} \partial_{a} \eta \partial_{b} \eta+\hat{R} \eta+\mu e^{\eta}\right)
$$

is the well known Liouville action. The induced integration measure in the $\eta$ space is then

$$
|\delta \eta|_{g}^{2} \approx \int d^{2} \sigma \sqrt{g}(\delta \eta)^{2}
$$


Assuming the validity of the DDK ansatz [15], we can express the Jacobian obtained in defining the measure with respect to the fiducial metric in terms of a renormalized Liouville action as follows

$$
\mathcal{D}_{g} \eta=\mathcal{D}_{\hat{g}} \eta \exp \left(\frac{1}{48 \pi} S_{L}\right)
$$

Thus, we obtain the following expression for the partition function

$$
Z=\int d^{2} \sigma \mathcal{D}_{\hat{g}} \eta \mathcal{D}_{\hat{g}} \phi_{i} \mathcal{D}_{\hat{g}} \vec{X} \mathcal{D}_{\hat{g}} b \mathcal{D}_{\hat{g}} c \exp \left(-S_{1}-S_{\vec{X}}-S_{\text {ghost }}\right),
$$

where $S_{\vec{X}}$ denotes the contribution of the $\vec{X}$ fields to the matter action (2) and

$$
\begin{aligned}
S_{1}= & \sum_{i} \int d^{2} \sigma \sqrt{\hat{g}}\left[\hat{g}^{a b}\left(\frac{D}{2} \partial_{a} \phi_{i} \partial_{b} \phi_{i}+B_{i} \partial_{a} \eta \partial_{b} \phi_{i}-\frac{A}{2} \partial_{a} \eta \partial_{b} \eta\right)\right. \\
& \left.-A \hat{R} \eta+B_{i} \hat{R} \phi_{i}+\mu e^{\eta}\right] .
\end{aligned}
$$

Here, $A=\frac{C+N-25}{12}$, and $D=1$, the usual normalization for free scalar fields. Assuming that the $U(1)$ 's are all realized at the same level in the Kac-Moody algebra, we can set the $B_{i}=B$ for all $i=1 \ldots N$, with $B$ left undetermined. Note that the total central charge of the matter sector is $c_{m}=C+N$.

The theory with the cosmological constant set to zero, $\mu=0$, is a conformal field theory with the stress tensor

$$
T(z)=\frac{A}{2} \partial_{z} \eta \partial_{z} \eta-A \partial_{z}^{2} \eta-\frac{D}{2} \partial_{z} \phi_{i} \partial_{z} \phi_{i}-\sum_{i} B_{i}\left(\partial_{z} \eta \partial_{z} \phi_{i}-\partial_{z}^{2} \phi_{i}\right) .
$$

Noting that the full quantum energy-momentum tensor can contain renormalized coupling constants other than those in the classical tensor derived from the action, we assume the most general form for the correlation functions for the $\eta-\phi_{i}$ system that is consistent with the equations of motion: 


$$
\begin{aligned}
<\eta(z) \eta(w)> & =\operatorname{alog}(z-w) \\
<\eta(z) \phi_{i}(w)> & =b_{i} \log (z-w) \\
<\phi_{i}(z) \phi_{j}(w)> & =\delta_{i j} d \log (z-w) .
\end{aligned}
$$

Computing the OPE of the energy-momentum tensor with itself, and assuming generic values of the coefficients given in eqns.(16) through (18), we get the central charge

$$
c_{i n t}=A a+D d+2 \sum_{i} B_{i} b_{i}+(N-1) D^{2} d^{2}-12 A,
$$

where the coefficients $a, b_{i}$ and $d$ are constrained by the equations

$$
\begin{aligned}
A & =A^{2} a+2 A \sum_{i} B_{i} b_{i}+\sum_{i} B_{i}^{2} d \\
D & =\sum_{i} B_{i}^{2} a+2 D \sum_{i} B_{i} b_{i}+D^{2} d \\
B_{i} & =B_{i} A a+\left(\sum_{i} B_{i}^{2}+A D\right) b_{i}+B_{i} D d .
\end{aligned}
$$

We also demand that the renormalized cosmological constant operator $e^{\alpha \eta}$ be a $(1,1)$ operator, so that $\int d^{2} \sigma \sqrt{\hat{g}} e^{\alpha \eta}$, which determines the surface area, stays invariant under a change of scale. Its conformal weight is given by

$$
\Delta(\alpha)=\frac{\alpha^{2}}{2}\left(a^{2} A+\sum_{i} b_{i}^{2} D+2 \sum_{i} B_{i} b_{i} a\right)+\alpha\left(A a+\sum_{i} B_{i} b_{i}\right) .
$$

From the requirement that the cosmological constant be primary, we infer that $A a+\sum_{i} B_{i} b_{i}=1$ and $D b_{i}+B_{i} a=0$. Using the system of equations for $a, b_{i}$ and $d$, we find that in general 


$$
\begin{aligned}
d & =\frac{A}{D A-\sum_{i} B_{i}^{2}} \\
b_{i} & =-\frac{B_{i}}{D A-\sum_{i} B_{i}^{2}} \\
a & =\frac{D}{D A-\sum_{i} B_{i}^{2}} .
\end{aligned}
$$

Using these relations it is easy to show that the central charge of the $\eta-\phi_{i}$ sector of the conformal field theory is given by

$$
c_{\text {int }}=2+(N-1) \frac{D^{2} A^{2}}{\left(D A-\sum_{i} B_{i}^{2}\right)^{2}} .
$$

and the conformal weight of the renormalized cosmological constant $: e^{\alpha \eta}:$ is

$$
\Delta(\alpha)=\frac{a^{2}}{2} \alpha^{2}+\alpha .
$$

Demanding that the theory be anomaly free, $c_{i n t}=26-C$, gives the constraint: either $N=1$, or $2 D A=N B^{2}$. If $N=1$, than $A=(C-24) / 12$, $D=1$, and $B$ is a free parameter. Upon choosing $B$ the values of the constants in the correlation functions (16)-(18) are given by the formulae (24)-(26). On the other hand if $N$ is kept arbitrary than $A=(C+N-25) / 12$, $D=1, B^{2}=-(C+N-25) / 6 N$, and again values for $a, b_{i}$ and $d$ follow from (24)-(26). Note that in both cases a well defined conformal field theory appears to exist on the world-sheet. Of course, if $B=0$, and we insist on having arbitrary $N$, it is not difficult to see that we are back to the usual DDK picture, with completely decoupled Liouville and matter sectors.

Collecting all of our results, we can now determine the possibilities for $\alpha$, the weight of the renormalized cosmological constant. The sign in the expression for $\alpha$ is chosen so as to agree with the semiclassical limit $c_{m} \rightarrow-\infty$ [21] [15]. 
(a) when $N=1$ and $B$ is arbitrary (also $D=-1$ and $A=(C-24) / 12$ )

$$
\alpha=\frac{1}{12}\left(24-C-12 B^{2}-\sqrt{\left(24-C-12 B^{2}\right)\left(-12 B^{2}-C\right)}\right) .
$$

with $\alpha$ real for $C \leq-12 B^{2}$, and $C \geq-12 B^{2}+24$,

(b) when $N B^{2}=2 D A, N \neq 1$, and $A=(C-25+N) / 12$, independent of the values of $B$ and $D$,

$$
\alpha=\frac{1}{12}(C-25+N-\sqrt{(C-25+N)(C-49+N)}) .
$$

In this case $\alpha$ is real for either $C+N \leq 25$ or $C+N \geq 49$. (For example, for the case of $S U(N)$ and $C=4$, we deduce that $N \leq 22$ or $N \geq 44$.)

In contrast to the case of matter fields of central charge $C$ coupled to twodimensional gravity, it seems that the renormalized cosmological constant operator is well behaved even for the case of physically interesting dimensions of the target space, namely $C=3,4$. The usual "negative world-sheet area" problem is apparently avoided.

\section{Scaling Laws}

In this section we discuss the scaling exponents of our model á la Knizhnik, Polyakov and Zamolodchikov [14] 15]. The partition function of our theory can be rewritten as

$$
Z=\sum_{\chi} \int_{0}^{\infty} d \mathcal{A} Z_{\chi}(\mathcal{A}),
$$

where $\chi=2(1-f)$ is the Euler characteristic, $f$ is the genus of the random surface, and $\mathcal{A}$ denotes its area. For large area, the partition fuction for fixed genus asymptotically behaves as [20]

$$
Z_{\chi}(\mathcal{A}) \approx \mathcal{A}^{b_{\chi}} \exp (-\kappa \mathcal{A})
$$


$\kappa$ being a renormalization dependent constant. It is natural therefore to consider the partition function for the case of fixed surface area, $\mathcal{A}$ (where for simplicity we do not consider integration over the moduli). Then,

$$
\begin{aligned}
Z(\mathcal{A})= & \int \mathcal{D}_{h} \eta \mathcal{D}_{h} \phi_{i} \mathcal{D}_{h} \Phi_{\text {matter }}^{0} \mathcal{D}_{h} b \mathcal{D}_{h} c \exp \left(-S_{\text {int }}-S_{\text {matter }}^{0}-S_{\text {gh }}\right) \\
& \delta\left(\int d^{2} \sigma \sqrt{h} e^{\alpha \eta}-\mathcal{A}\right) .
\end{aligned}
$$

The string susceptibility, $\gamma_{s t r}$, is usually defined as $b_{\chi}=\gamma_{s t r}-3$ [14, thus

$$
Z(\mathcal{A}) \approx \mathcal{A}^{\gamma_{s t r}-3}
$$

We will now compute this scaling exponent. Note the invariance of the original partition function under $\hat{g}^{a b} \rightarrow \hat{g}^{a b} e^{-\omega}$ and $\eta \rightarrow \eta+\frac{\omega}{\alpha}$, where $\omega$ is an arbitrary constant [15]. The action rescales as (thus far, we have left the genus of the surface arbitrary)

$$
S \rightarrow S-A(1-f) \frac{\omega}{\alpha} .
$$

Then

$$
\delta\left(\int d^{2} \sigma \sqrt{h} e^{\alpha \eta}-\mathcal{A}\right) \rightarrow e^{-\omega} \delta\left(\int d^{2} \sigma \sqrt{h} e^{\alpha \eta}-e^{-\omega} \mathcal{A}\right) .
$$

and we obtain the following scaling law:

$$
Z(\mathcal{A}) \rightarrow \mathcal{A}\left(-1+(1-f) \frac{A}{\alpha}\right)
$$

leading to the following special cases: (a) if $N=1$

$$
\gamma_{s t r}=2+(1-f) \frac{C-24}{24-C-12 B^{2}-\sqrt{\left(24-C-12 B^{2}\right)\left(-12 B^{2}-C\right)}}
$$

(b) if $N$ is different from one

$$
\gamma_{s t r}=2+(1-f) \frac{C-25+N}{C-25+N-\sqrt{(C-25+N)(C-49+N)}} .
$$


Notice that here the familiar problem of complex scaling exponents for $C>1$ does not arise. It would be tempting at this point to conclude that smooth surfaces exist in our model even if the dimension of the target space is greater than unity as long as $C+N \leq 25$, with the world-sheet theory a well defined conformal field theory. We postpone the criticism of this naive conclusion until the next section.

One can also compute the gravitational dressing of the operators, or the change in the operator's scaling dimensions in the background of the fluctuating two-dimensional metric. Let $\Phi$ be a spinless primary field in the matter theory with the conformal weight $\Delta_{0}=\Delta_{0}(\Phi)=\bar{\Delta}_{0}(\Phi)$. The corresponding gravitationally dressed operator is $\Phi e^{\beta \eta}$. In order for

$$
\int d^{2} \sigma \sqrt{h} \Phi e^{\beta \eta}
$$

to make sense, $\Phi e^{\beta \eta}$ has to be a $(1,1)$ operator or in other words the following requirement

$$
\Delta_{0}+\frac{a}{2} \beta^{2}+\beta=1
$$

has to be satisfied. We conclude that

$$
\beta=\frac{1}{12}\left(C-25+N \pm \sqrt{(C-25+N)\left(C-49+N-24 \Delta_{0}\right)}\right) .
$$

Furthermore the gravitational scaling dimension $\Delta(\Phi)$ can be deduced from [14], [15]

$$
Z_{\Phi}(\mathcal{A}) \approx \mathcal{A}^{1-\Delta}
$$

where $Z_{\Phi}(\mathcal{A})$ is the expectation value of the one-point function

$$
Z_{\Phi}(\mathcal{A})=\frac{\int \mathcal{D}_{h} \Psi e^{-S} \delta\left(\int d^{2} \sigma \sqrt{h} e^{\alpha \eta}-\mathcal{A}\right) \int d^{2} \sigma \sqrt{h} \Phi e^{\beta \eta}}{Z(\mathcal{A})} .
$$


In the last expression we have collectively denoted both matter and ghosts by $\Psi$. S denotes the complete action of matter and ghost fields. Using the same scaling argument as before we get

$$
\Delta=-\frac{\beta}{\alpha}
$$

implying that the gravitational scaling dimension satisfies the following equation

$$
\Delta-\Delta_{0}=\frac{a}{2} \alpha^{2} \Delta(\Delta-1),
$$

and we recall that $a$ was defined by (26). Again, if we set $B=0$ and use (26)-(28) we obtain the familiar result of [14] [15].

Finally we discuss the mean square size and the corresponding Hausdorff dimension of random surfaces defined by our theory, computed in [22 for Polyakov's string (the case $B=0$ ). We closely follow their treatment. As before we are interested in the limit of very large area, $\mathcal{A} \rightarrow \infty$. Then the Hausdorff dimension $d_{H}$ of the surface is defined by looking at its mean square size, namely

$$
<X^{2}>_{\mathcal{A}} \approx C \mathcal{A}^{2 / d_{H}}
$$

where $C$ denotes the dimension of target space. This critical exponent 23] provides a measure of the interaction in the physical system under consideration. If the dimensionality of target space is greater than twice the Hausdorff dimension then the system is essentially free. If on the other hand, the dimensionality of target space is less or equal to the Hausdorff dimension, the interactions play a crucial role in determining the physics of the system.

We now consider the Fourier transform of the "point-split" version of the above definition of the mean square size following [22], to extract the value 
of $d_{H}$

$$
G\left(k^{2}\right)=<\int d^{2} \sigma_{1} d^{2} \sigma_{2} \sqrt{g\left(\sigma_{1}\right)} \sqrt{g\left(\sigma_{2}\right)} \exp \left(i k\left(X\left(\sigma_{1}\right)-X\left(\sigma_{2}\right)\right)>_{\mathcal{A}}\right.
$$

It follows that

$$
<X^{2}>_{\mathcal{A}}=C\left|2 \partial_{k^{2}} \log G\left(k^{2}\right)\right|_{k=0}
$$

Using the fact that the vertex operator $V_{k}=\exp (i k X)$ is gravitationally dressed, and that in the zero momentum limit it reduces to the cosmological constant operator, we write

$$
G\left(k^{2}\right)=<\int d^{2} \sigma_{1} d^{2} \sigma_{2} \sqrt{\hat{g}\left(\sigma_{1}\right)} \sqrt{\hat{g}\left(\sigma_{2}\right)} \tilde{V}_{k} \tilde{V}_{-k}>_{\mathcal{A}}
$$

where

$$
\tilde{V}_{k}=\exp (i k X)\left(a\left(k^{2}\right) \exp \left(\beta_{+} \eta\right)+b\left(k^{2}\right) \exp \left(\beta_{-} \eta\right)\right),
$$

with $\beta_{ \pm}$denoting the positive and negative roots of $(42)$, and $a(0)=1$, $b(0)=0$. After some algebra,

$$
<X^{2}>=C\left(K_{1}-M \log \mathcal{A}+K_{2} \mathcal{A}^{\left|\gamma_{s t r}^{s p h}\right|}\right)
$$

where $\gamma_{s t r}^{s p h}$ is the string susceptibility, given by formulae (38) and (39) for spherical topology $(f=0)$, and

$$
M=1+\sqrt{\frac{49-C-N}{25-C-N}} .
$$

Hence $d_{H}=\gamma_{s t r}^{s p h} / 2$. We omit the corresponding (long!) expressions for $K_{1}$ and $K_{2}$, except to note that $M, K_{1}$ and $K_{2}$ are all positive, providing the positivity of the mean sqaure size of the random surface. 


\section{Discussion}

In this section we point out the shortcomings of this model and summarize what we have learned from this exercise. Let us start with the form of the interaction term. The reader has undoubtedly noticed that this term ex-

plicitly breaks the translation invariance of the $\phi_{i}$ sector, thereby rendering $\phi_{i}$ noncompact, as is the Liouville field in the familiar Polyakov string. We could analytically continue the $\phi_{i}$ fields to imaginary values (thus imposing the analog of the background charge selection rules familiar from the treatment of the Liouville field) by integrating over the constant mode of $\phi_{i}$. The background charge would be proportional to the value of the coupling constant $B$. As with Liouville theory, it would also be necessary to to introduce screening operators in the correlators to absorb the residual momenta.

We should note that modifications similar to our interaction term have already appeared in the string theory literature within the context of the Polyakov string. Myers [24] has considered a linear dilaton background given by $n_{\mu} X^{\mu}$ where $n_{\mu}$ is a fixed space-time vector. In this particular example the space-time Lorentz invariance is spoiled, even though the central charge of the matter fields (the critical dimension) is arbitrary. Unlike our proposal above, no interaction term is induced since Myers' action is defined with respect to the fiducial metric, and the Liouville field has of course decoupled.

More recently Kawai and Nakayama [26] have considered a similar interaction term, but in their case $\phi$ is a massive auxiliary degree of freedom which, on being integrated out, generates an $R^{2}$ correction to the Einstein action. We also note that our model defines an explicit Lagrangian that will generate the form of the stress tensor proposed by Cohn and Periwal [27] 
provided that we interpret our fields $\phi_{i}$ as the additional ghosts in their picture. And indeed the matter Lagrangian defined with respect to the fiducial metric $\hat{g}$ has the general form of the bosonized ghost Lagrangians [28]. The $D=1$ barrier is also apparently evaded in the chiral gravity theory of [29]. Finally, we point out that the diagonalized form of our energy momentum tensor (apart from peculiar field dependent coupling constants) has the same form as the energy-momentum tensor for the set of "Liouville-like" scalar fields that appear in the bosonic construction of $W_{N}$ theories [30]). If appropriate linear combinations of the $\phi_{i}$ and the Liouville field are introduced such that the resulting holomorphic energy-momentum tensor does not contain the interaction term, then the field dependence is shifted to the coupling constants, one of them being the world-sheet cosmological constant.

However, as is well known, the above mentioned procedure of analytic continuation leads to another grave difficulty, namely, the instability of the vacuum state. As was noted by Myers in the case of the critical string with the linear dilaton background, a single string would tend to split into two strings carrying complex momenta and therefore imply a ground state energy unbounded from below. A similar problem was encountered by Natsuume [25] in a nonlinear sigma approach to the effective string theory proposed by Polchinski and Strominger. Our calculations of the critical exponents are, therefore, purely formal in nature, the ground state being unstable.

Our aim has been to illustrate through an example (unphysical, as it turned out) that the induced gravity sector of the Polyakov string can be consistently modified such that the usual indicators of the onset of the "strong gravity" regime, namely the various scaling exponents we have discused above, all look perfectly reasonable even for target spaces of physically interesting dimensionality. This example, we believe, illustrates a possibly 
useful direction to examine in constructing realistic non-critical string theories.

\section{Acknowledgements}

We thank J. Cohn, R. Myers, and J.Polchinski for useful comments on the manuscript. This work is supported by the Robert A. Welch Foundation, and by NSF grant PHY 9009850. 


\section{References}

[1] A.Neveu, in Recent Advances in Field Theory and Statistical Mechanics, ed. by J.-B.Zuber and R.Stora (Elsevier) 1984.

[2] J.Polchinski, preprint UTTG-16-92, (1992).

[3] D.J.Gross, preprints PUPT-1355 and PUPT-1356 (1992).

[4] D.C.Lewellen, Nucl.Phys. B392 (1993) 137, and refences therein.

[5] K.G.Wilson, Phys.Rev. D10 (1974) 2445;

D.Weingarten, Phys.Lett. B90 (1980) 280, 285;

V.A.Kazakov, Phys.Lett. B128 (1983) 316;

I.K.Kostov, Phys.Lett. B138 (1984) 191;

K.H.O'Brien and J.-B.Zuber, Nucl.Phys. B253 (1985) 621;

I.K.Kostov, Nucl.Phys. B265 (1986) 223.

[6] G.'t Hooft, Nucl.Phys. B72 (1974) 461;

E.Witten, Nucl.Phys. B160 (1979) 57.

[7] G.'t Hooft, Nucl.Phys B75 (1974) 461;

C.G.Callan, N.Coote and D.J.Gross, Phys.Rev. D13 (1975) 1649;

A.A.Migdal, Sov.Phys.JETP 42 (1975) 413;

V.A.Kazakov and I.K.Kostov, Nucl.Phys. B176 (1980) 199;

B.Rusakov, Mod.Phys.Lett. A5 (1990) 693.

[8] The pioneering work is S.Mandelstam, Ann.Phys. (N.Y.) 19 (1962)

1. For a comprehensive review consult A.A.Migdal, Phys. Rep. 102C (1983) 199, and references therein.

[9] D. Gross and W. Taylor, preprints PUPT-1376/LBL-33458, and PUPT1382/LBL33767 (1993);

J.Minahan, Virginia preprint, UVA-HEP-92010 (1993);

J. Minahan and A. Polychronakos, hep-th/9303153 (1993);

M. Douglas, Rutgers preprint, RU-93-13 (1993);

I.K.Kostov, Saclay preprint SPhT/93-050 (1993).

[10] S.R.Das, S.Naik and S.R.Wadia, Mod.Phys.Lett. A4 (1989) 1033;

T.Banks and J.Lykken, Nucl.Phys. B331 (1990) 173;

J.Polchinski, Nucl.Phys. B346 (1990) 253.

[11] J.Polchinski and A.Strominger, Phys.Rev.Lett. 67 (1991) 1681.

[12] T.Banks, Rutgers University preprint, RU-90-52, (1990).

[13] A.M.Polyakov, Phys.Lett. B103 (1981) 207;211. 
[14] V. Knizhnik, A. Polyakov and A. Zamalodchikov, Mod. Phys. Lett. A (1988).

[15] F.David, Mod.Phys.Lett. A3 (1988) 1651;

J.Distler and H.Kawai, Nucl.Phys. B321 (1988) 509.

[16] J.Polchinski, Phys.Rev.Lett. 68 (1992) 1267.

[17] A.M.Polyakov, Phys.Lett B82 (1979) 247; Nucl.Phys. B164 (1979) 171; Gauge Fields and Strings, Harwood Academic, 1987.

[18] A. M. Polyakov, Nucl. Phys. B268 (1986) 406; also in Fields, Strings and Critical Phenomena, ed. by E.Brézin and J.Zinn-Justin (Elsevier) 1989 ;

P. B. Weigmann, Nucl. Phys. B323 (1989) 311.

[19] For a review, see P. Goddard and D. Olive, in Vertex Operators in Mathematics and Physics, ed. by J. Lepowsky, S. Mandelstam and I. M. Singer (Springer Verlag) 1984.

[20] T. Eguchi and H. Kawai, Phys. Lett. B110 (1982) 143, B114 (1982) 247.

[21] A.B.Zamolodchikov, Phys.Lett. B117 (1982) 87; S.Chaudhuri, H.Kawai and S.-H.Henry Tye, Phys.Rev.D36 (1987) 1148.

[22] J.Distler, Z.Hloušek and H.Kawai, Int.Journ.Mod.Phys. A5 (1990) 1093.

[23] G.Parisi, Phys.Lett. B81 (1979) 357.

[24] R. Myers, Phys. Lett. B199 (1988) 371.

[25] M.Natsuume, Texas preprint, UTTG-10-92, June 1992.

[26] H.Kawai and Nakayama, KEK-TH-355, KEK preprint 92-212, UT-634, February 1993.

[27] J.D.Cohn and V.Periwal, Phys.Lett. B270 (1991) 18.

[28] D. Friedan, E. Martinec and S. Shenker, Nucl. Phys. B271 (1986) 93.

[29] R. Myers and V. Periwal, Nucl. Phys. B397 (1993) 239.

[30] For a review, see C.M.Hull, QMW/ph/92/1, (1992);

S.R.Das, A.Dhar and S.K.Rama, Tata preprints, TIFR/TH/91-11, TIFR/TH/91-20 (1991). 